\title{
Perioperative complications of the modified Stoppa approach for the treatment of pelvic bone fractures: a single-institution review of 48 cases
}

\author{
Hyeonjoon Lee, MD (iD, Suenghwan Jo, MD, PhD iㅣ, Gwangchul Lee, MD, PhD (iD, Yongjin Cho, MD, PhD (iD) \\ Department of Orthopedic Surgery, Chosun University Hospital, Chosun University College of Medicine, Gwangju, Korea
}

Received: August 9, 2021

Revised: October 18, 2021

Accepted: November 1, 2021

Correspondence to

Yongjin Cho, MD

Department of Orthopedic Surgery,

Chosun University Hospital, Chosun

University College of Medicine, 365

Pilmun-daero, Dong-gu, Gwangju

61453, Korea

Tel: +82-62-220-3147

E-mail: choisidoru@chosun.ac.kr
Purpose: The current study aimed to report the perioperative complications of the modified Stoppa approach for the treatment of pelvic bone fractures.

Methods: We analyzed 48 consecutive operations in 45 patients who were treated with internal fixation using the modified Stoppa approach between March 2016 and July 2018. This included three revision operations. The mean age of the patients was 54.5 years, and the patients included 35 male patients and 10 female patients. All fractures occurred as a consequence of high-energy trauma and $70.3 \%$ had associated injuries at the time of the fracture. The mean Injury Severity Score was $9.03 \pm 5.60$. The perioperative complications found during and immediately after surgery were recorded and were classified into three categories: vascular injuries, nerve injuries, and other complications.

Results: Overall, 14 perioperative complications (29.2\%) in 14 cases were identified. The most common complications were nerve injuries, which occurred in seven cases, all involving the obturator nerve. Uncontrollable vascular injuries occurred in six cases, which required additional incisions and support of vascular surgeons or postoperative interventions. Additionally, one case of peritoneal tearing occurred, which required help from an abdominal surgeon.

Conclusions: While the modified Stoppa approach seems to be a viable method to treat pelvic fractures, significant perioperative complications may occur, suggesting that surgeons should pay careful attention to minimize the damage to other structures and that appropriate support from other surgical departments is paramount.

Keywords: Pelvis; Acetabulum; Fracture; Modified Stoppa approach; Perioperative complication

\section{INTRODUCTION}

Pelvic fractures with acetabular involvement occur at the weight-bearing joints and are best treated through open reduc- tion and internal fixation. Anatomic reduction with stable column fixation is required to provide optimal outcomes [1].

However, a surgical approach to the fractured pelvis is difficult because of its sophisticated anatomical structure and its

(C) 2022 The Korean Society of Traumatology

This is an Open Access article distributed under the terms of the Creative Commons Attribution Non-Commercial License (http://creativecommons.org/licenses/by-nc/4.0/) which permits unrestricted non-commercial use, distribution, and reproduction in any medium, provided the original work is properly cited. 
deep location [2]. A currently used approach for the treatment of acetabular fractures is the modified Stoppa approach, which was described by Hirvensalo et al. [3] in 1993 and Cole and Bolhofner [4] in 1994. Compared to the present ilioinguinal approach, the modified Stoppa approach can be done with a smaller incision and with minimal dissection of the pelvic structures. Operations using the modified Stoppa approach are mostly successful. However, the potential complications during the operation, such as vascular and nerve injuries, may be a significant concern, especially for novice surgeons. Nonetheless, there are limited reports on the perioperative complications associated with the modified Stoppa approach. Herein, we report our experiences of perioperative complications using the modified Stoppa approach.

\section{METHODS}

This study was approved by the Institutional Review Board of the Chosun University Hospital (No. 2021-06-033). A retrospective study of 48 operations in 45 patients using the modified Stoppa approach between March 2016 and July 2018 was conducted. Using hospital records and radiographic examinations, the following data were collected: demographics, Injury Severity Score, the mechanism of injury, the presence of associated injuries, and perioperative complications. The demographic data of these patients are listed in Table 1. Operations were performed by a single surgeon specializing in hip and pelvis fractures. The quality of the reduction was not evaluated

Table 1. Demographic data $(n=45)$

\begin{tabular}{lc}
\hline Variable & Value \\
\hline Age (yr) & $54.50 \pm 15.39$ \\
Sex & \\
$\quad$ Male & $35(77.8)$ \\
$\quad$ Female & $10(22.2)$ \\
Injury mechanism & \\
$\quad$ Fall down & $24(53.3)$ \\
Traffic accident & $17(37.8)$ \\
Compression injury & $4(8.9)$ \\
Injury Severity Score & $9.03 \pm 5.60$ \\
Pelvic fracture without acetabular involvement & $3(6.7)$ \\
$\quad$ Stoppa approach only & $2(66.7)$ \\
Additional approach (Kocher-Langenbeck) & $1(33.3)$ \\
Pelvic fracture with acetabular involvement & $42(93.3)$ \\
$\quad$ Stoppa approach only & $36(85.7)$ \\
$\quad$ Additional approach (Kocher-Langenbeck) & $6(14.3)$ \\
\hline
\end{tabular}

Values are presented as mean \pm standard deviation or number (\%). in this study because the correlations with clinical outcomes have already been well-described and the aim of the present study was to identify the perioperative complications [5].

\section{Surgical methods}

All operations were performed using the method described previously. The first case was operated using a Deaver retractor, while the other 47 cases were operated with a carbon precurved retractor designed for the modified Stoppa approach. Eleven cases used a conventional reconstruction plate, while 37 cases used a precontoured anatomical plate designed specifically for medial buttress fixation (Stryker, Kalamazoo, MI, USA). During surgery, the obturator nerve and vessels were identified using the vessel loops to protect them from further damage. If we encountered vascular anastomoses between the external iliac vessels and obturator vessels (i.e., corona mortis), they were dissected and ligated using vascular clips. According to the fracture patterns, the Kocher-Langenbeck approach was also performed posteriorly in seven patients. One of those seven patients had a pelvic fracture without an acetabular-involved fracture pattern, and six of the seven patients had pelvic fractures showing acetabular involvement. Reconstruction plates (Synthes, Solothurn, Switzerland) contoured to patients' specific pelvic bony structures were used.

\section{Records of complications}

All complications were recorded by the surgeon immediately after surgery. The complications were categorized as vascular injuries, nerve-related injuries, and other complications. Vascular injuries were defined as uncontrolled bleeding that required additional incision, postoperative embolization, or assistance from a vascular surgeon.

A nerve-related injury was defined as any alteration or deficiency of motor or sensory function that was not observed prior to surgery. Symptoms of numbness, adductor muscle weakness or neuropathy, and any condition diagnosed with an electrodiagnostic test were included. The occurrence of neurological symptoms was evaluated during outpatient postoperative follow-up after surgery considering the possibility that the surgeons might not have been aware of neurological complications during surgery. Postoperative complications such as post-traumatic osteoarthritis and infection were not evaluated in this study.

\section{RESULTS}

Overall, there were 14 perioperative complications: seven cases 
of nerve injuries, six cases of vascular injuries, and one case of peritoneal injury (Table 2).

All seven cases of nerve-related injuries involved the obturator nerve (Fig. 1). The patients with nerve-related symptoms complained of numbness and some degree of tingling. As there were no cases of iatrogenic nerve disruption during surgery, it was assumed that the nerve-related symptoms may have occurred due to traction of the nerve during plate insertion or direct compression by the medial buttress plate. Of the six cases of vascular injuries, two were external iliac vein injuries that occurred during revision surgery. The external iliac vein was repaired with the help of vascular surgeons. One case involved rupture of a branch of the internal iliac artery. In that case, an additional incision was made for exploration and ligation was performed by a vascular surgeon. Another two cases involved abrupt bleeding deep in the sciatic notch. The bleeding was controlled by gauze packing followed by clipping of the vessels. While the exact location of the bleeding was unidentified, it was assumed to occur in vascular branches of the superior gluteal artery. One other case was related to the epigastric artery and occurred during Hemovac insertion (Fig. 2). This injury was controlled with a radiologic intervention. It should be noted that four of the six cases of vascular injuries occurred in pa- tients who had previous abdominal surgery, such as a cesarean section or peritonitis surgery.

In addition to the nerve- and vascular-related complications, one case of peritoneal tearing occurred (Fig. 3). The patient had a history of abdominal surgery because of previous trauma. Due to severe adhesions during the approach, the anatomical structure of the abdominal wall was not easily distinguished. The peritoneal tear occurred during the dissection, and an area of the small intestine was observed in the operative field. A general surgeon investigated the possibility of internal organ damage and confirmed that no additional damage was present; subsequently, peritoneal repair was performed.

In the 48 operations, there were three revision operations. Two cases were performed because of unsatisfactory reductions and one case was performed because of nonunion. In all three cases, we tried to achieve anatomical reduction and perform refixation using a plate without additional procedures.

\section{DISCUSSION}

Treatment of pelvic ring injuries and acetabular fractures is very challenging for trauma surgeons, and especially orthopedic surgeons. Since these fractures are caused by high-energy

Table 2. Perioperative complications $(n=48)$

\begin{tabular}{lcl}
\hline Subtype & Complication (\%) & \\
\hline Vascular injury & $6(12.5)$ & 2 External iliac vein, 1 internal iliac artery branch, 1 superior gluteal artery, 1 epigastric artery \\
Nerve injury & $7(15.6)$ & Obturator nerve \\
Other complications & $1(2.1)$ & Peritoneum tear \\
\hline
\end{tabular}
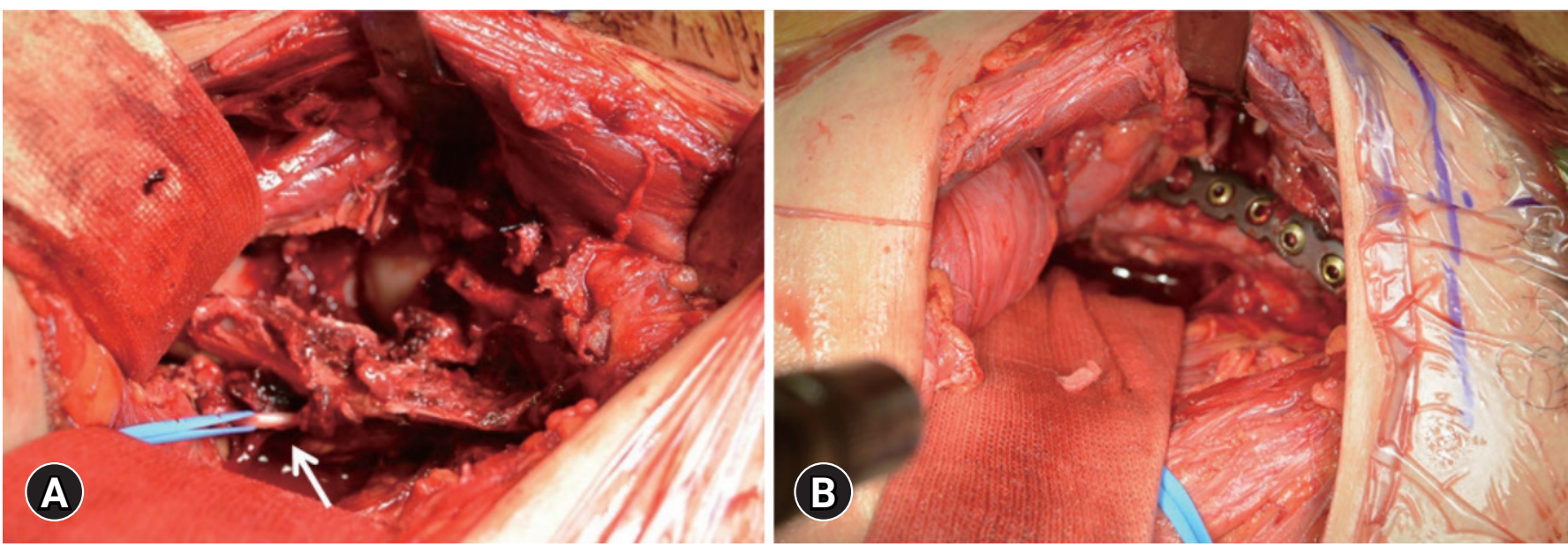

Fig. 1. (A) Obturator nerve lies cross the iliopectineal line (arrow). (B) The obturator nerve can be injured during initial injury or reduction of the fracture site, so attention must be needed. The patient provided written informed consent for publication of the research details and clinical images. 

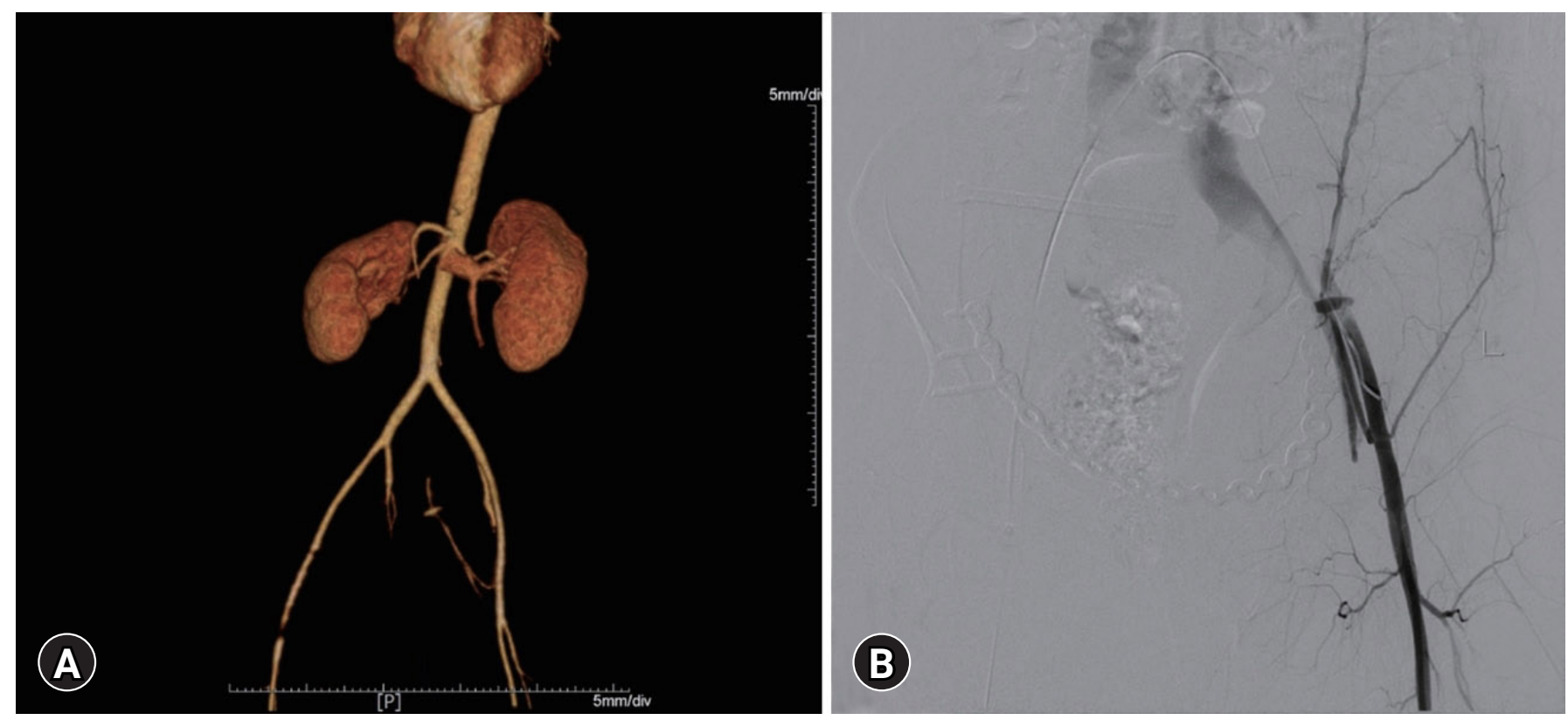

Fig. 2. Left epigastric artery pseudoaneurysm following the modified Stoppa approach. (A) Three-dimensional computed tomography angiography. (B) Angiography. The patient provided written informed consent for publication of the research details and clinical images.

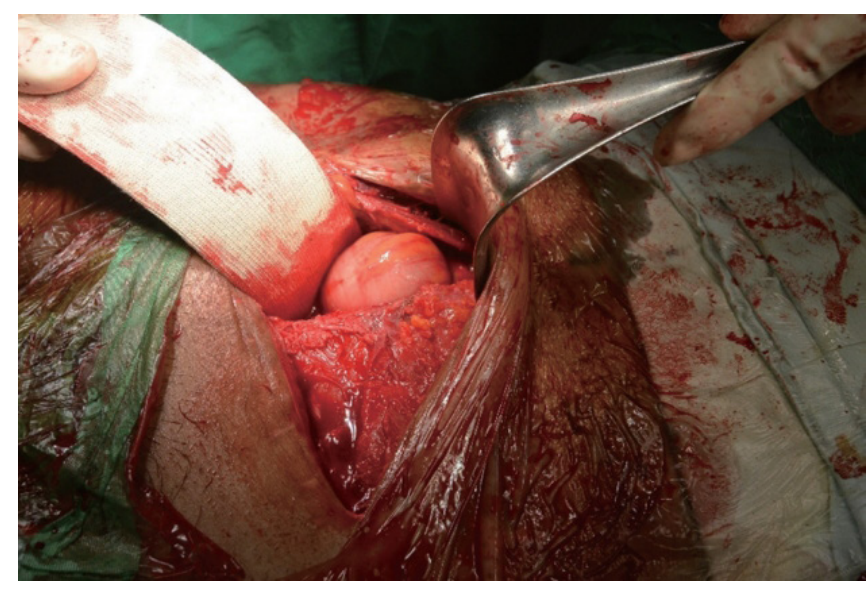

Fig. 3. A peritoneal tear occurred during the modified Stoppa approach. The patient had a history of previous abdominal surgery. The patient provided written informed consent for publication of the research details and clinical images.

trauma, which is accompanied by damage to internal organs and blood vessels, collaboration with several departments may be needed to treat these patients.

The modified Stoppa approach is very useful for the treatment of acetabular fractures and pelvic ring injuries. It can help to access and visualize the displaced quadrilateral surface, directly reduce the medially displaced quadrilateral surface, and easily insert a buttress plate into the inferior pelvic brim through only a single incision. It also enables direct reduction of the im- pacted acetabular dome. However, it has some disadvantages. For instance, the obturator nerve can be injured during exposure of the quadrilateral surface. In cases of posterior column fractures, the screw insertion angle is limited. A hernia may occur postoperatively. The complications of the modified Stoppa approach are known to include external iliac vein injuries, obturator nerve injuries, sciatic nerve palsy, wound infection, and fixation failure. Other complications have also been reported, such as inguinal hernia, violation of the peritoneal cavity, cystotomy, lateral femoral cutaneous nerve palsy, superior gluteal artery, atrophy of the rectus abdominis muscle, and deep vein thrombosis $[4,6,7]$.

In this study, we experienced a total of 14 perioperative complications. Obturator nerve injuries were the most common, in seven cases (50.0\%), followed by vascular injuries in six cases (42.9\%), and peritoneal tearing in one case (7.1\%).

Vascular injuries are among the most fatal complications that can occur during the modified Stoppa approach. The authors experienced six vascular injuries. In four out of the six cases, the patients had a history of previous abdominal surgery (e.g., cesarean section and peritonitis surgery). The damaged vessels were the external iliac vein in two cases (33.3\%), a branch of the internal iliac artery in one case (16.7\%), and bleeding from deep in the sciatic notch in two cases (branches of the inferior gluteal artery or inferior epigastric artery) (33.3\%). According to Archdeacon et al. [7], the most common complications related 
to vessels associated with the modified Stoppa approach are external iliac vein injury, while another complication of note is superior gluteal artery injury. Soni et al. [8] described injuries of the corona mortis, obturator artery, external vein, and superior gluteal artery. In our experience, the corona mortis was found in 27 cases (57.4\%). In several cases, the corona mortis was injured in the initial trauma.

If surgeons use the modified Stoppa approach, they must pay attention to ways of reducing the complications related to vascular injuries. First, retraction of the anterior abdominal wall elements causes tension on the femoral vessels. The femoral vessels and nerve can be damaged by direct or traction injuries during retraction of the anterior abdominal elements. In elderly patients, the femoral vessels are particularly friable and susceptible to retraction [9]. Second, the obturator vessels or nerve can be damaged during exposure of the quadrilateral plate or by incorrect application of a medial plate in the quadrilateral plate. In the first third of dissection of the pelvic brim, the anastomotic branches between the internal and external iliac systems are described as the corona mortis. Although it was reported in $49.3 \%$ of cases, the corona mortis varies in terms of its size and surgical significance [10]. In all cases, the anastomotic vessels need to be dissected and ligated. Third, injuries to the superior gluteal vessels, for which bleeding control is difficult, can take place during careless retraction or reduction. Fourth, another obstacle is posed by the nutrient vessel branches from the iliolumbar artery. Before elevation of the posterior iliacus, it can be clipped at the internal iliac artery to prevent excessive bleeding. We recommend the following to surgeons unfamiliar with the modified Stoppa approach as ways to reduce these vascular complications and respond appropriately: (1) preparing sufficient blood, (2) starting with compression, (3) using a vascular clip that is kept handy, and (4) performing the operation during regular hours.

The obturator nerve is one of the most important structures that should be considered during intrapelvic approaches. The prevalence of the accessory obturator nerve is reported to be between $0 \%$ and $29 \%$, and obturator nerve injuries occurred in $14.6 \%$ of cases in our study. Other studies have reported that the incidence of obturator nerve injuries associated with the modified Stoppa approach ranged from $0 \%$ to $26 \%[4,11-13]$. However, those studies did not distinguish between traumatic and iatrogenic injuries. In contrast, Isaacson et al. [14] and Kim et al. [15] reported no complications of obturator nerve injury using the modified Stoppa approach. The course of the obturator nerve originates from the obturator canal and lies across the iliopectineal line. The course of this nerve makes it vulnerable to injury. There are two main mechanisms by which the obturator nerve can be damaged. First, it can be injured in initial trauma, which is particularly related to the extent of displacement of the quadrilateral plate. Kim et al. [15] reported that the prevalence of obturator nerve injuries from the initial trauma was $9 \%$ in their series of cases using the modified Stoppa approach, and these injuries were related to the extent of displacement of the quadrilateral plate ( $>24 \mathrm{~mm}$ ). Second, it can be injured intraoperatively. The obturator nerve passes through the obturator canal as it crosses the iliopectineal line, and the risk of injury increases during plate application or when using various instruments for reduction (e.g., a collinear clamp) [16]. The nerve was distracted only by $13.1 \mathrm{~mm}$ from the iliopectineal line via retraction [17]. To reduce the risk of obturator nerve injury, surgeons should recognize the possibility of obturator nerve injury in the initial trauma and explore and release the obturator nerve using the modified Stoppa approach. When reducing the quadrilateral lateral plate, if using reduction tools (e.g., a collinear clamp) it is necessary to check the entrapment of the obturator nerve at the fracture site, and care must be taken not to damage the nerve when fixing the plate and holding the retractors.

Regarding other complications, we experienced one case of peritoneal tearing. The patient had a history of previous abdominal surgery, and the peritoneal tear is thought to have occurred in this patient due to adhesions in the peritoneum resulting from previous surgery. Another study mentioned the possibility of damage to the peritoneal cavity as a disadvantage of the modified Stoppa approach [18]. In order to reduce these unexpected injuries, in the preoperative planning stage, if the patient has a history of gynecological or urological surgery, it should be recognized that there is a risk of perforation of bladder and infection after surgery. In particular, in patients with a history of previous prostatectomy or radiotherapy, the risk of bleeding during the approach is high, so the surgeon should consider other surgical approaches as a way to reduce complications that may occur during surgery [7]. Other contraindications include abdominal distension and ileus [4].

Limitations of this study include the small number of study subjects and the inability to analyze the factors for the occurrence of various complications due to the limited applicability of statistical methods. However, this was the first report describing perioperative complications that occurred during the modified Stoppa approach performed by a single surgeon at a single institution, and it is expected that these findings will be 
helpful for surgeons performing the modified Stoppa approach for the first time.

In conclusions, injury to the obturator nerve was the most common nerve-related injury, and care should be taken when inserting the retractor and performing plate fixation. Vascular injuries and unexpected injuries can be fatal to the patient. Therefore, the authors recommend that the surgeons should pay careful attention when operating on patients at high risk of complications (e.g., with previous abdominal procedures, old age, and other risk factors) using the modified Stoppa approach.

\section{NOTES}

\section{Ethical statement}

This study was approved by the Institutional Review Board of the Chosun University Hospital (No. 2021-06-033). Due to the retrospective nature of this study, the Institutional Review Board waived consent.

\section{Conflicts of interest}

The authors have no conflicts of interest to declare.

\section{Funding}

None.

\section{Author contributions}

Conceptualization: CYJ, JSH; Data curation: LHJ, JSH; Investigation: LHJ; Supervision: CYJ; Writing-original draft: LHJ, JSH; Writing-review\&editing: LGC.

All authors read and approved the final copy of the manuscript.

\section{REFERENCES}

1. Borg T, Berg P, Larsson S. Quality of life after operative fixation of displaced acetabular fractures. J Orthop Trauma 2012;26:445-50.

2. Kim HY, Yang DS, Park CK, Choy WS. Modified Stoppa approach for surgical treatment of acetabular fracture. Clin Orthop Surg 2015;7:29-38.

3. Hirvensalo E, Lindahl J, Bostman O. A new approach to the internal fixation of unstable pelvic fractures. Clin Orthop Relat Res 1993;(297):28-32.

4. Cole JD, Bolhofner BR. Acetabular fracture fixation via a modified Stoppa limited intrapelvic approach. Description of operative technique and preliminary treatment results. Clin Orthop Relat Res 1994;(305):112-23.
5. Giannoudis PV, Grotz MR, Papakostidis C, Dinopoulos H. Operative treatment of displaced fractures of the acetabulum. A meta-analysis. J Bone Joint Surg Br 2005;87:2-9.

6. Jakob M, Droeser R, Zobrist R, Messmer P, Regazzoni P. A less invasive anterior intrapelvic approach for the treatment of acetabular fractures and pelvic ring injuries. J Trauma 2006;60:1364-70.

7. Archdeacon MT, Kazemi N, Guy P, Sagi HC. The modified Stoppa approach for acetabular fracture. J Am Acad Orthop Surg 2011;19:170-5.

8. Soni A, Gupta R, Sen R. Modified Stoppa approach for acetabulum fracture: a review. Rev Bras Ortop (Sao Paulo) 2019; 54:109-17.

9. Khoury A, Weill Y, Mosheiff R. The Stoppa approach for acetabular fracture. Oper Orthop Traumatol 2012;24:439-48.

10. Sanna B, Henry BM, Vikse J, et al. The prevalence and morphology of the corona mortis (Crown of death): a meta-analysis with implications in abdominal wall and pelvic surgery. Injury 2018;49:302-8.

11. Laflamme GY, Hebert-Davies J, Rouleau D, Benoit B, Leduc S. Internal fixation of osteopenic acetabular fractures involving the quadrilateral plate. Injury 2011;42:1130-4.

12. Hammad AS, El-Khadrawe TA. Accuracy of reduction and early clinical outcome in acetabular fractures treated by the standard ilio-inguinal versus the Stoppa/iliac approaches. Injury 2015;46:320-6.

13. Elmadag M, Guzel Y, Aksoy Y, Arazi M. Surgical treatment of displaced acetabular fractures using a modified Stoppa approach. Orthopedics 2016;39:e340-5.

14. Isaacson MJ, Taylor BC, French BG, Poka A. Treatment of acetabulum fractures through the modified Stoppa approach: strategies and outcomes. Clin Orthop Relat Res 2014; 472:3345-52.

15. Kim JW, Shon HC, Park JH. Injury of the obturator nerve in the modified Stoppa approach for acetabular fractures. Orthop Traumatol Surg Res 2017;103:639-44.

16. Kacra BK, Arazi M, Cicekcibasi AE, Buyukmumcu M, Demirci S. Modified medial Stoppa approach for acetabular fractures: an anatomic study. J Trauma 2011;71:1340-4.

17. Locher S, Burmeister H, Bohlen T, et al. Obturator nerve block: a technique based on anatomical findings and MRI analysis. Pain Med 2008;9:1012-15.

18. Meena S, Sharma PK, Mittal S, Sharma J, Chowdhury B. Modified Stoppa approach versus ilioinguinal approach for anterior acetabular fractures: a systematic review and meta-analysis. Bull Emerg Trauma 2017;5:6-12. 